\title{
Kitting versus line stocking: A conceptual framework and a descriptive model*
}

\author{
Yavuz A. Bozer ${ }^{\mathrm{a}}$ and Leon F. McGinnis ${ }^{\mathrm{b}}$ \\ ${ }^{a}$ Department of Industrial and Operations Engineering, The University of Michigan, Ann Arbor, MI 48109, USA \\ ${ }^{\mathrm{h}}$ School of Industrial and Systems Engineering, Georgia Institute of Technology, Atlanta, GA 30332, USA
}

(Received 3 March 1991; accepted in revised form 15 March 1992)

\begin{abstract}
In this paper, based on a number of site visits we performed, we present certain fundamental differences between kitting and line stocking (i.e., no kitting), and we develop definitions which are intended to serve as a framework to study most kitting operations. We also develop a descriptive model which can be used to quantify the trade-offs in material handling, space requirements, and work-in-process between kitting and line stocking at an early decision stage. We present a numerical example to demonstrate the model and some of the insight we gained. Lastly, we discuss new research directions to further explore kitting and its impact on the shop floor.
\end{abstract}

\section{Introduction}

In manufacturing systems, the practice of delivering components and subassemblies to the shop floor in predetermined quantities that are placed together in specific containers is generally known as "kitting". Although we will formally define a kit in the next Section, for discussion purposes a kit may be generally vicwed as a container which holds a specific assortment of parts that are used in one or more assembly operations in the plant. Rather than delivering the required parts to an assembly station in component containers and in relatively large quantities, parts can be first

Correspondence to: L.F. McGinnis, Material Handling Research Center, Georgia Institute of Technology, 765 Ferst Drive, NW, Atlanta, GA 30332-0205, USA.

* This article was originally accepted by the Journal of Manufacturing and Operations Management. pulled together in "kit containers" (and sometimes temporarily stored) before they are delivered to the shop floor. Thus, with kitting, no part inventories are kept at the assembly stations (other than "expendable" parts that are usually not included in any kit).

For example, a major manufacturer of electronics and telecommunications equipment delivers the required parts for their electronic switching circuits in kits. The assembly stations are arranged along a straight line conveyor (which resembles a transporter) and a number of boards are assembled concurrently. A majority of the parts are delivered in kits which are prepared in a stockroom located in a nearby mezzanine. The parts required for the kits are picked on a "walk-and-pick" basis where each worker prepares several kits on each trip through the stockroom. The components are stored in bins and the bins are placed on shelves. Completed kits are issued from the stockroom and manually delivered to 
the line by a tractor/trailer system as they are needed. Except for a short holding period in the stockroom, there is no intermediate storage for completed kits, i.e., they are delivered directly to the line.

A leading manufacturer of personal computers, on the other hand, uses a slightly specialized approach. The primary components (such as the case, the drives, the printed circuit boards, the power supply, etc.) are stored in gravity flow racks. These components are moved (usually in pallet loads) from the receiving area to the back end of the flow racks where they are placed in appropriate lanes. The kit preparation stations are located in the front end of the flow racks. The workers in this area pull the parts from the flow racks and arrange them on a special-purpose tray which holds the case and all the primary components. Each worker is assigned a dedicated zone along a conveyor which holds the tray as the kit is being prepared. Also, each worker prepares only part of the kit which is moved from one zone on the conveyor to the next as the kit is prepared. Once it is ready, the kit - which resembles a "loosely assembled" personal computer - is delivered via roller conveyor to one of the assembly stations. Since the components are placed in their proper positions in the case, the time required to assemble a unit is reduced. In fact, the kit needs to visit only one of a dozen or so parallel assembly stations where the assembly operations are performed prior to testing and burn-in.

Kitting applications similar to the ones described above have been used for a reasonably long time and in a variety of settings in industry. However, as part of an overall operational strategy, kitting is still not well understood and it remains somewhat controversial. Proponents of kitting point out that it gives the user better control over work-in-process (WIP) - through increased parts visibility and accountability on the floor - and that it helps reduce manufacturing floor space, while opponents claim that the labor consumed in assembling the kits is nonproductive labor (since it is not a "value added" operation) and that kitting is used primarily to "conceal" poor manufacturing operations management.

The debate continues at least partly due to the fact that no model that can be used to study the impact of kitting has been developed. Although some trade journals contain brief descriptions of specific applications of kitting (see for example Refs. $[1,2]$ ), to our knowledge, no general purpose model that would facilitate the selection process - between kitting, partial kitting, and line stocking (i.e., no kitting) - has been reported in the literature.

A study reported by Wilhelm and Wang [3] is concerned with the inventory management aspect of kitting and the timing of the kit assembly process. The authors present mathematical/statistical models that describe kit earliness, kit tardiness, and in-process time for component inventory. (In-process time is defined as the sum of the time the component waits in the component storage area and the time it waits in the kit storage area as part of an "early kit".) The authors use these models to describe "fundamental characteristics of the (kitling) process such as the inherent relationship between expected (kit assembly) time and the number of required components." The study concentrates primarily on "component accumulation" (i.e., the kit assembly operation) and it does not include the material handling aspects of kitting such as container slorage and retrieval and container flow in the facility.

Another study concerned with kitting is presented by Sellers and Nof [4] who conducted a survey on the status of kitting in industry by polling 120 US manufacturing firms representing over 15 industries. Their survey indicates that "kitting is an established practice in assembly industries". According to the survey "the overall most-frequent kitting configuration found in the survey ( 5 cases) has kits produced at staging (kit assembly) areas and then transported primarily by roller conveyors to the assembly cells". The authors also report that "typically, companies... are kitting nearer to the (component) storage facility, rather than kitting closer to the assembly area, and are picking parts manually and directly 
from bins". (The reader may refer to the article for further details.) Sellers and Nof also present a qualitative comparison of robotic versus manual kitting. For robotic kitting, the authors describe several system design configurations based on equipment such as carousels, automated storage/retrieval systems, and automated guided vehicles.

Since there are no general purpose models reported in the literature on line stocking versus kitting, and since kitting is primarily an industrial problem, early in study we visited a number of installations to observe first-hand several applications of kitting in industry and to discuss various aspects of kitting with the users. We also had several discussions with individuals from industry who, mostly for reasons cited earlier, did not support kitting. The material presented in the next two Sections is largely a result of this effort.

\section{Definitions and major assumptions}

We will define a component as a fabricated or purchased part that cannot be subdivided into distinct constituent parts. For example, sheet metal is a component for a stamping plant. A subassembly is the aggregation of two or more components and/or other subassemblies through an assembly process. The end product is the result of a series of assembly operations which requires no further processing in the current facility. For example, an engine is an end product for an engine plant, but a component for an automobile assembly plant. A stock keeping unit (i.e., SKU number) is used to designate any item that is stored and/or handled in the facility; the item can be a component, a subassembly, or an end product. Each item has a unique SKU number.

A kit is a specific collection of components and/or subassemblies that together (i.e., in the same container) support one or more assembly operations for a given product or "shop order." Each kit type is assigned a unique kit number. The type of components and subassemblies required for each kit type (along with the number of pieces required of each) is given by the kit structure. A container which contains only one component type, say, container $A$, will qualify as a kit if the corresponding component container is retrieved from storage and a specified quantity of the component is transferred to container $A$ (which has been assigned to a particular product or shop order).

A kit container is assumed to hold only one kit type, but it is allowed to hold multiple kits of the same type. Kits per container is an integer number that designates the number of kits in a kit container for each kit type. Obviously, by changing the kit structure one may have two kits of type A or one kit of type B in a kit container. That is, both kit types would require identical component types but one would have twice the number of pieces as the other for each component type. Kit assembly is an operation where all the components and/or subassemblies that are required for a particular kit type are physically placed (sometimes in specific positions) in the appropriate kit container. Conceptually, kit assembly is an order picking operation. As such, alternative methods exist for assembling kits. In order to perform a quantitative comparison between kitting and line stocking, we will assume that the kit assembly operation is based on "end-ofaisle" order picking. That is, the appropriate component containers are retrieved and brought to the end of the storage aisle where the appropriate number of parts are removed from each container.

In most cases, since several component and/or subassembly containers must be retrieved to assemble a kit, it is fairly common to assemble several kits of the same type simultaneously. That is, once a component or subassembly container is brought to the kit assembly area, one may pick enough pieces from that container to assemble several kits of a given type. After the required parts are retrieved, the component or subassembly container is returned to storage (provided the container is not empty). The number of kits (of the same type) that are assembled simultaneaously as described above is defined as the kit batch size. 
Based on the site visits we performed and our discussions, certain characteristics of industrial kitting operations emerged as follows. (The sites we visited and the industrial companies with which we interacted were mostly represented by the electronics and/or computer industry. The primary process was an assembly process.) First, there are mainly two types of kitting operations: kit-to-customer and kit-to-manufacturing. The former is concerned with pulling the appropriate parts together prior to shipping them to the customer. For example, certain ready-to-assemble kits that are available through the mail for electronic products would fall into this category.

The latter category, kit-to-manufacturing, is concerned with pulling the required parts together in kit containers which are subsequently delivered to the shop floor to support one or more assembly operations. As evidenced by the two examples we presented earlier, this study is concerned with kit-tomanufacturing. (A third type of kitting occurs when a maintenance person prepares a kit of spare parts and supplies before he/she is dispatched to the service location. The main issue in such applications is to determine the number and type of spare parts and supplies to include in the maintenance kit. In this paper we will not address maintenance kits.) The remainder of this Section and the paper are devoted to kit-to-manufacturing applications, in particular those encountered in assembly operations.

In our site visits, we observed two types of kits: stationary kits and traveling kits. A stationary kit is delivered to a workstation and it remains there until it is depleted. The product to be assembled moves from one workstation to another (independent of the stationary kit(s)). A traveling kit is handled along with the product and it supports several workstations before it is depleted. There are two types of traveling kits. The first type is a single container where the kit and the product travel in the same container as the product is assembled. With the second type, the product travels in one container (or fixture) while the kit follows the product in parallel in another con- tainer. The two travel together from one workstation to another. (The traveling kit of the second type was one of the alternatives considered by a major automotive manufacturer to provide certain parts needed at each assembly station along with the automobile body.)

Regardless of the type, however, a kit typically does not contain all the parts required to assemble one unit of the end product. This is sometimes due to product complexity or product size. Also, certain components such as fasteners, washers, etc. are almost never included in kits; instead, such parts are bulk delivered to the shop floor in component containers. We also observed that most applications use a number of kit containers in various types and sizes to accommodate different kit types. Regardless of the kit container types, the shop configuration and the process plan together usually dictate the kit structure and the delivery points for stationary kits. In most cases, however, kitting appears to generate additional material handling due to kit delivery and return of empty kit containers. This is not necessarily the case as we will later demonstrate with an example.

As far as application scenarios are concerned, there are two distinct scenarios. In the first one, there is a uniform kit mix, i.e., the daily mix of end products and the corresponding kits are reasonably stable. In this casc, a justin-time (JIT) approach to kitting may be practical. With JIT kitting, the kits are assembled on demand and sent directly to the assembly stations. With a uniform kit mix it is relatively straightforward to identify the "average day" in order to predict the demands placed on kit assembly.

In the second scenario, there is a variable kit mix, i.e., the mix of end products being assembled varies significantly from day to day (or week to week). For example, there might be several hundred product types, but on any one day, only a few, say, 50-100, would be assembled. In this scenario, it would be more difficult to achieve a JIT kitting operation since the kit assembly workload can vary significantly and it may not be proportional to the assembly 
workload. Thus, kitting in advance and kit storage may become necessary. This creates two difficulties from an analysis viewpoint. First, it becomes more difficult to identify an "average day" for purposes of setting the kit assembly capacity (and for purposes of comparing kitting with line stocking). Second, in addition to component storage and retrieval, one must analyze the storage and retrieval of completed kits. This would require further input such as an estimate of the average kitting lead time, i.e., how far in advance must a batch of kits be assembled.

The model we present accommodates both scenarios described above, provided that the decision-maker is willing or able to identify the "worst day" case for the variable kit mix and that JIT kitting is used. The latter implies that we do not address kit container storage and retrieval since we assume that all the kit containers, once they are assembled, are directly dispatched to the appropriate workstation on the shop floor. (Note that another operational issue - which we do not address in this study - that becomes more evident with the variable kit mix is the assignment of on-hand inventory to kits planned for assembly.)

\section{Kitting: advantages and limitations}

The following list represents the advantages of assembling kits and delivering a majority of the parts to the shop floor in kit containers:

(1) Saves manufacturing space and reduces work-in-process at the workstations by storing primary components and subassemblies at a central storage area.

(2) Since the majority of components and subassemblies are not staged at the workstations, product changeover is accomplished with relative ease.

(3) Offers better control and higher flexibility by handling and routing only the kit containers through the assembly system (as opposed to a wide array of individual component containers).
(4) Facilitates material delivery to workstations by eliminating the need to supply individual component containers.

(5) Provides better control and visibility for high cost and/or perishable components and subassemblies.

(6) Offers potential increase in product quality and workstation productivity since the parts are readily available and/or pre-positioned. In some cases, a kit may actually resemble a "loosely assembled" product.

(7) Supports small batch size operations with a large variety of products mainly because it facilitates product changeover.

(8) Facilitates robotic handling at the workstations by presenting an opportunity (during kit assembly) to control the exact quantity, position, and orientation of individual parts placed in the kit container.

Limitations of kitting can be presented as follows:

(1) Kit preparation (i.e., kit assembly) consumes time and effort (usually in the form of manual labor) with little or no direct value added to the product.

(2) Is likely to increase storage space requirements, especially when kits are prepared in advance.

(3) Demands additional planning to assign on-hand parts to kits, especially when kits contain several common components.

(4) Temporary shortage of parts may force the user to kit short; doing so will reduce the overall efficiency of the operation (due to the double-handling of the kit containers and the additional storage space required by partially assembled kits).

(5) Defective parts that are inadvertently used in certain kits will lead to parts shortages at the workstations. Kits that contain defective parts must be "reassembled."

(6) Components that may fail during (or as a result of) the assembly process, will require special consideration or exceptions (i.e., they may have to be excluded from the kits). One may be forced to provide either a spare piece with each kit or to store 
component containers at some workstations.

(7) If parts shortages develop (due to defective parts or other reasons), some kits may get "cannibalized". That is, short parts may be removed from some of the existing kits. This may further complicate the shortage and it may lead to problems in parts accountability. Also, it will almost always lead to double-handling - first to remove the short part from existing kits and later to add the part to "cannibalized" kits when a new shipment is received.

Some of the limitations of kitting are mostly eliminated when the kits are not assembled far in advance; that is, when the kits are assembled on a JIT basis with minimal inventories of completed kits. However, as we noted earlier, with a variable kit mix, JIT kitting may not be feasible.

Although we do not claim the above lists to be exhaustive, they reflect primary arguments for and against kitting, and they are mostly consistent with the lists presented by Sellers and Nof [4]. In our view, kitting does seem to be an appropriate approach in certain cases. However, given the wide range and diversity of existing assembly operations, the challenge is to identify those cases which seem most suitable for kitting. The descriptive model we developed as part of this study is aimed at formulating and quantifying certain differences that exist between kitting and line stocking to facilitate the above decision. (Here line stocking refers to the case where all the components are delivered to the work-stations in component containers.) Before we present this descriptive model, however, in the following Section we will present the problem environment.

\section{Kitting operations}

\subsection{General material flow}

Figure 1 depicts the conceptual flow of material in assembly systems with kitting.
(A similar representation is also reported in Ref. [4].) The components received from outside the system are stored in a storage area where they remain until they are needed. Note that some components, following component preparation, may be directly delivered to the production area while other components are first assembled in kits in a kit assembly area. Subsequently, completed kits are either stored in a kit storage area or delivered directly to the production area. After the components are combined with one another to form subassemblies they will remain in the production area until they are either put back in storage to be used in other kits or they leave the system as end products.

The storage areas shown in Fig. 1 are not necessarily physically separate areas. In some applications it is not unusual to store completed kits in the same system along with components and subassemblies. Also, in some applications (primarily in the electronics industry), the component preparation area and the kit assembly area are the same areas.

\subsection{Relation to other subsystems}

Setting up and operating a kitting system is a complicated task since kitting interacts with many subsystems normally present in assembly operations. For example, the appropriate kit structure depends on the bill-of-material and the process plan. Furthermore, the number and type of kits assembled during particular time periods (and the corresponding kit batch sizes) depend on the production volumes and production batch sizes planned for the end products.

In many instances, kitting also affects component, subassembly, and WIP storage in the facility. If the kits are assembled ahead of time, kit storage/retrieval itself requires additional planning. A closely related issue is container selection. The type and number of component and subassembly containers required may vary dramatically between kitting and line stocking. The kit container, on the other hand, is closely related to the kit structure. The latter, combined with the production schedule, 


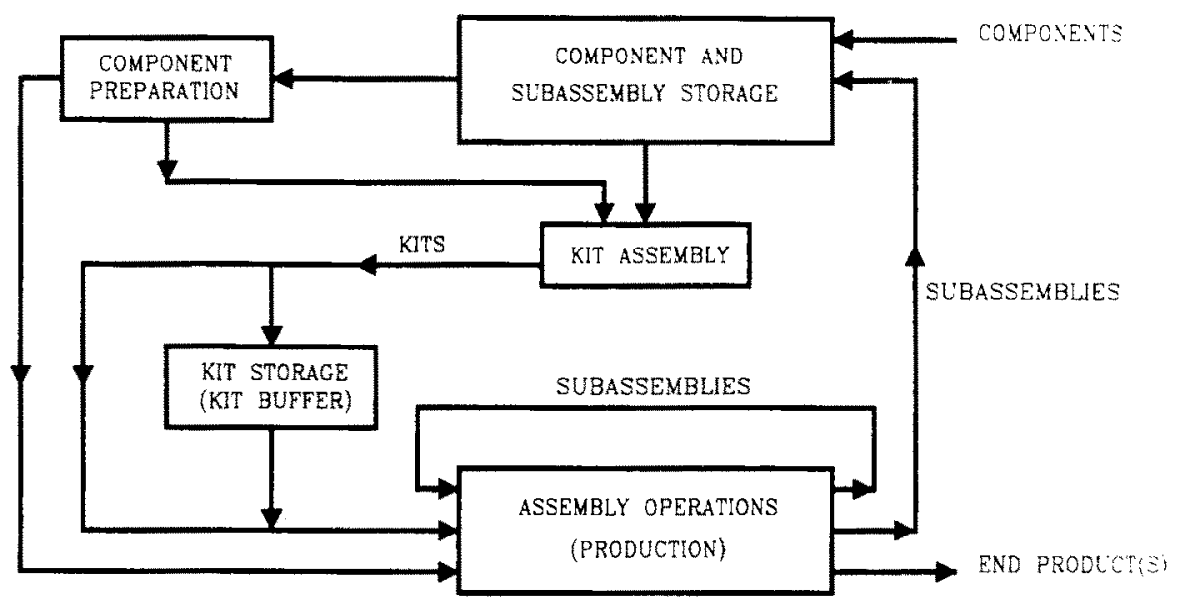

Fig. 1. General material flow in kitting-based assembly operations.

dictates how and when the kits are assembled and delivered to the assembly area. (Designing the kit assembly operation itself could be as complicated as designing the assembly area.) Lastly, the layout of the facility, the material handling system, and the shop floor control mechanism may vary significantly between kitting and line stocking. In general, the above interactions are likely to show certain variations even between alternative kitting plans.

\section{A descriptive model}

As seen from the previous Section, kitting is tightly coupled with other subsystems and decision mechanisms that support a manufacturing operation. Developing a comprehensive model that would capture all of the above interactions seems not only far from straightforward but premature. The modeling effort is further complicated by the fact that a majority of the above interactions, their nature, their significance, and the appropriate way to model them seems to vary considerably from one application to another.

That is, in a practical sense, it is difficult to separate issues related to kitting from the rest of the system and study them in isolation. However, as shown in this Section, if certain simplifying assumptions are made, some of the trade-offs involved between kitting and line stocking can be quantified. In particular, the model we present is concerned with identifying the material handling trade-offs between kitting and line stocking. We will examine the difference between the two concepts relative to container storage and retrieval as well as overall container flow within the facility. We also show the resulting floor space requirements and average WIP levels under simple line stocking or kit delivery rules.

We will first present our notation and modeling assumptions, followed by the corresponding expressions of interest. In the next Section, we will use a numerical example to demonstrate the model and to discuss the insight we gain from it. For ease of exposition, we assume that there is a single component container type and a single kit container type. We also assume that there is only one type of end product assembled. Of course, the model can be extended to multiple container types and multiple end products by adding the appropriate subscripts. Let:

$s$ denote the SKU number for a component, subassembly, or end product,

$p_{s}$ denote the number of pieces of SKU $s$ stored in a component container, $m_{s}$ denote the number of pieces of SKU $s$ used in one unit of the end product, 
$w$ denote the workstation type,

$\pi_{w}$ denote the number of parallel (i.e., identical) workstations of type $w$,

$a_{w}$ denote the floor space required by workstation $w$ (not including space required for staging component or kit containers),

$D$ denote the number of end products produced in an $8 \mathrm{~h}$ day,

$\bar{W}$ denote the average WIP expressed as the average number of partially assembled products in the system,

$\Phi_{w}$. denote the set of SKU numbers used at workstation $w$,

$\alpha_{s w}$ denote the number of component containers of SKU $s$ staged at each workstation of type $w$,

$F$ denote the floor space required to stage a component container at a workstation.

To model the impact of kitting, we need the following additional notation. Let:

$k$ denote the kit type $k$,

$p_{\text {sk }}$ denote the number of pieces of SKU $s$ used in kit type $k$,

$d_{k}$ denote the number of kits of type $k$ needed to produce one unit of the end product; note that $d_{k}=m_{s} / p_{s k}$,

$b_{k}$ denote the batch size for kit type $k$; i.e., the (integer) number of type $k$ kits assembled when a component container is retrieved,

$q_{k}$ denote the (integer) number of kit containers per batch for kit type $k$,

$n_{k}$ denote the number of kits per kit container for kit type $k$; note that $b_{k}=q_{k} n_{k}$,

$S_{k}$ denote the set of SKU numbers assigned to kit type $k$,

$\Omega_{w}^{\prime}$. denote the set of all stationary kit types staged at workstation lype $w$,

$\beta_{k w}^{\prime}$ denote the number of stationary kit containers of type $k \in \Omega_{w}^{\prime}$ staged at each workstation of type $w$,

$\Omega^{\prime \prime}$ denote the set of traveling kit types that travel along with the product,

$\beta_{k}^{\prime \prime}$ denote the number of traveling kit containers of type $k \in \Omega^{\prime \prime}$ that travel along with the product,

$f$ denote the floor space required to stage a stationary kit container at a workstation.
Unless otherwise stated, a kit may be a stationary kit or a traveling kit. Also, note that $d_{k}$ may or may not be an integer number; its value depends on the kit structure. Assuming that all of the above information is given, we will determine the values of the following variables which form the basis of comparison between line stocking and kitting:

$c_{w}$ is the total number of component containers to be supplied to all the workstations of type $w$ per $8 \mathrm{~h}$ day under line stocking,

$R$ is the number of component containers that must be stored or retrieved per day to support production; $R$ is expressed in number of "operations" per day where one operation is a container retrieval or container storage,

$V$ is the number of kit or component containers per day that flow from the storage/kit assembly area to all the workstations, $A_{w}$ is the floor space required by all the workstations of type $w$ (including the space required by component and kit containers),

$A$ is the total floor space required by all the workstations (not including aisle space and other clearances),

$h_{w}$ is the average WIP level at workstation $w$ in number of component pieces,

$H$ is the average WIP level for all the workstations in number of component pieces.

Remaining assumptions concerned with the model are presented as follows:

(1) With line stocking, each workstation that uses SKU $s$ is supplied with a component container of SKU s. That is, workstations do not share component containers. (Although in some applications container sharing might be possible or desirable, in most assembly operations each workstation is supplied with its own component containers.)

(2) We assume that $p_{s} \gg b_{k} p_{s k}$ for all $s$ and $k$. That is, the number of pieces in a component container of SKU $s$ is considerably larger than the number of pieces of SKU $s$ pulled from the component container each time it is retrieved to assemble a batch of kit type $k$. 
(3) We will consider only the flow of component (or stationary kit) containers from the storage (or kit assembly) area to the workstations. That is, we will not address the flow of the product itself (and the associated traveling kits, if any) since we assume that product flow is not affected by how the workstations are supplied by components.

(4) In computing $V$, we will not consider empty component container return. If the empty component containers are not stackable, one can multiply our results by two to account for their return to the storage area. The same assumption applies to empty kit containers.

(5) WIP is divided into two categories: WIP due to component (or stationary kit) containers, and WIP due to partially assembled products. Although we will use the average number of partially assembled products in the system, i.e., $\bar{W}$, to compute the WIP due to traveling kits, we will primarily focus on the former category of WIP because it is likely to vary dramatically between line stocking and kitting. Unless kitting has a significant impact on the mean or the distribution of the workstation cycle times, $\bar{W}$ will not vary considerably between line stocking and kitting.

(6) For line stocking we assume that a new component container is delivered only when the current one is near depleted. (This assumption is supported by the justin-time principle.) We also assume that, once it is delivered to the shop floor, the contents of a component container are consumed uniformly over time. The same assumptions apply to stationary kit containers.

(7) We assume that the contents of a traveling kit container decrease uniformly over time as the corresponding product travels through the shop.

Based on the above assumptions, in the next four subsections we derive expressions to estimate container handling, floor space requirements, and average WIP under line stocking and kitting.

\subsection{Component container storage and retrieval}

We will first present the rate at which component containers must be stored and retrieved under line stocking. With line stocking, each component container must be retrieved from storage and dispatched to the appropriate workstation. Hence, the total number of component containers per day to be supplied to all the workstations of type $w$, that is, $c_{w}$, can be obtained from the following expression:

$c_{w^{\prime}}=\sum_{s \in \Phi_{w}}\left(D m_{s}\right) / p_{s}$.

Note that, in Eq. (1), we show all the containers retrieved for all the workstations of a particular type; we do not indicate the containers retrieved for individual workstations since each workstation of the same type uses the same number of containers, by definition.

In the long term, since each component container that is retrieved must be eventually replenished (i.e., stored), the number of component containers which must be stored or retrieved per day, that is, $R$ is given by

$R=2 \sum_{w} c_{w}$

operations/day for systems with line stocking.

If kitting is used, the rate at which component containers are retrieved depends on several factors. Recall that $\left|S_{k}\right|$ denotes the number of SKU types assigned to kit type $k$ and $b_{k}$ denotes the kit batch size (i.e., the number of kits assembled from a component container each time it is retrieved). Hence, to assemble one kit of type $k$, we would need to retrieve $\left|S_{k}\right|$ component containers. However, we may assemble more than one kit (of the same type) once we retrieve the appropriate component container. Since we need $d_{k}$ kits per end product, to assemble a total of $b_{k}$ kits per container retrieval, we would need to retrieve a component container $\left(D d_{k}\right) / b_{k}$ times per day. Note that this expression is also equal to the number of batches of kits assembled per day, by definition. (Of course, $b_{k}$ must be integer divisible by 
$n_{k}$ since the number of kit containers assembled per batch, $q_{k}$, is an integer number.)

Hence, with kitting, the rate at which component containers must be stored or retrieved, that is, $R$ is given by

$$
R=\left(2 \sum_{k} \frac{D d_{k}}{b_{k}}\left|S_{k}\right|\right)+\sum_{w^{*}} c_{w},
$$

where the first term is multiplied by two since every component container which is retrieved while assembling a kit must, by definition, be stored back in the storage system after the required parts are pulled from the container. The second term in Eq. (3) represents the rate at which the containers must be stored in the system as part of the replenishment cycle. Note that, due to the second term, Eq. (3) yields the approximate number of operations performed per day. In reality, following kit assembly, since an empty container will not be placed back in storage, some of the replenishment operations can be "interleaved" with the retrieval operations.

\subsection{Component and kit container flow}

In comparing line stocking with kitting, we are also concerned with the amount of container flow generated by the two alternatives. (Recall that we will not address the product flow and the flow associated with traveling kits, if any.) With line stocking, all the component containers retrieved from the storage area are dispatched to the appropriate workstation. Hence, the number of (component) containers per day that flow from the storage area to all the workstations, $V$, is given by the following expression:

$V=\sum_{w} c_{w}$,

where $c_{w}$ is given by Eq. (1).

If kitting is used, the number of (stationary) kit containers per day that flow from the storage/kit assembly area to all the worksta- tions is given by the following expression:

$$
V=\sum_{w} \sum_{k \in \Omega_{w}^{\prime}} \frac{D d_{k}}{n_{k}}
$$

Recall that, in computing $V$, we do not consider empty component (or kit) container return.

\subsection{Shop floor space requirements}

Consider first line stocking. Recall that each workstation of type $w$ needs enough space to accommodate $\alpha_{S w}$ component containers for each type of SKU used. Thus,

$$
\begin{aligned}
& A_{w^{\prime}}=\pi_{w^{\prime}}\left(a_{w}+F \sum_{s \in \Phi_{w}} \alpha_{s w^{\prime}}\right), \\
& A=\sum_{w} A_{w^{\prime}},
\end{aligned}
$$

where $A_{w}$ is the total floor space required by all the workstations of type $w$ and $A$ represents the total floor space required for the shop. The above floor space requirement does not include an aisle space allowance since it would not be likely to change between line stocking and kitting.

Consider next kitting. Since a workstation needs enough space to accommodate $\beta_{k w}^{\prime}$ containers of (stationary) kit type $k$, we have

$A_{w}=\pi_{w}\left(a_{w}+f \sum_{k \in \Omega_{w}^{\prime}} \beta_{k w}^{\prime}\right)$.

The total floor space required by the workstations, $A$, is still given by Eq. (7). If a workstation uses only traveling kits, i.e., no (stationary) kits are staged at the workstation, then we will set $f=0$ to obtain $A_{w}=\pi_{w} a_{w}$ for that particular workstation type.

\subsection{Work-in-process}

The WIP due to component (or stationary kit) containers is mostly determined by the replenishment method used to supply the 
workstations. Given our just-in-time assumption for container deliveries, and a uniform consumption of components, with line stocking the average WIP at the workstations and the total average WIP in the system (measured in number of pieces) are given by

$$
\begin{aligned}
& h_{w}=\pi_{w} \sum_{s \in \Phi_{w}} \frac{1}{2} \alpha_{s w} p_{s}, \\
& H=\sum_{w} h_{w^{\prime}}
\end{aligned}
$$

If kitting is used, the average WIP due to stationary kits, measured in number of pieces, can be obtained from the following expressions:

$$
\begin{aligned}
& h_{w}=\frac{1}{2} \pi_{w} \sum_{k \in \Omega_{w}^{\prime}} \beta_{k w}^{\prime}, n_{k} \sum_{s \in S_{k}} p_{s k}, \\
& H=\sum_{w} h_{w} .
\end{aligned}
$$

Since traveling kits move along with the product, the WIP due to traveling kits is not straightforward to determine. That is, the number of traveling kits in the system is a function of the number of partially assembled products in the system. Furthermore, as the product moves through the assembly operations, more components in the corresponding traveling kit become part of the product. The following approach, however, is likely to yield a reasonable approximation of WIP due to traveling kits.

The number of pieces of SKU $s$ in one kit container for kit type $k$ is equal to $n_{k} p_{s k}$, and the total number of pieces in a kit container for kit type $k$ is equal to $n_{k} \sum_{s \in S_{k}} p_{s k}$. Hence, as one unit of the product approaches the first workstation, the total number of pieces in the corresponding traveling kits is equal to $\sum_{k \in \Omega^{\prime \prime}} \beta_{k}^{\prime \prime} n_{k} \sum_{s \in S_{k}} p_{s k}$.

The average WIP in pieces, $H$, depends on how the components in a traveling kit are consumed as they travel through the shop. (It also depends on the delay encountered in traveling from one workstation to another; note that $\bar{W}$ captures this delay.) Given our uniform consumption assumption, we have

$$
H=\frac{1}{2} \bar{W}\left(\sum_{k \in \Omega^{\prime \prime}} \beta_{k}^{\prime \prime} n_{k} \sum_{s \in S_{k}} p_{s k}\right) .
$$

Note that some traveling kits may be consumed before the others. This may violate our "uniform consumption" assumption. However, if all the traveling pieces are viewed collectively, and if the delay from one workstation to another is distributed uniformly across the system, it seems reasonable to assume that the total piece count decreases uniformly.

Also note that, if most or all the traveling kits are consumed before the product reaches the last workstation, our approach will overestimate the average WIP due to traveling kits. However, one can adjust the value of $\bar{W}$ by determining the last workstation where the traveling kit was used. The average number of partially assembled products between the first workstation and this last workstation would be the appropriate value to use for $\bar{W}$.

\section{A numerical example}

We will demonstrate the model and some of the trade-offs between line stocking and kitting using a numerical example based on a hypothetical assembly operation. The product to be assembled is a stationary fitness cycle. The exploded assembly drawing of the end product is shown in Fig. 2. The corresponding parts list is shown in Table 1 where the SKU number is listed as the "part number." For brevity, we will use the "key number" shown in Table 1 as the SKU number. The number of pieces of SKU $s$ per end product, $m_{s}$, is also shown in Table 1 under the column labeled "Quantity".

We assume the frame (SKU \#1) is never delivered in a component container or a kit container. Instead, each frame is moved, one at a time, through six types of assembly stations $(w=1, \ldots, 6)$. Each workstation type is 


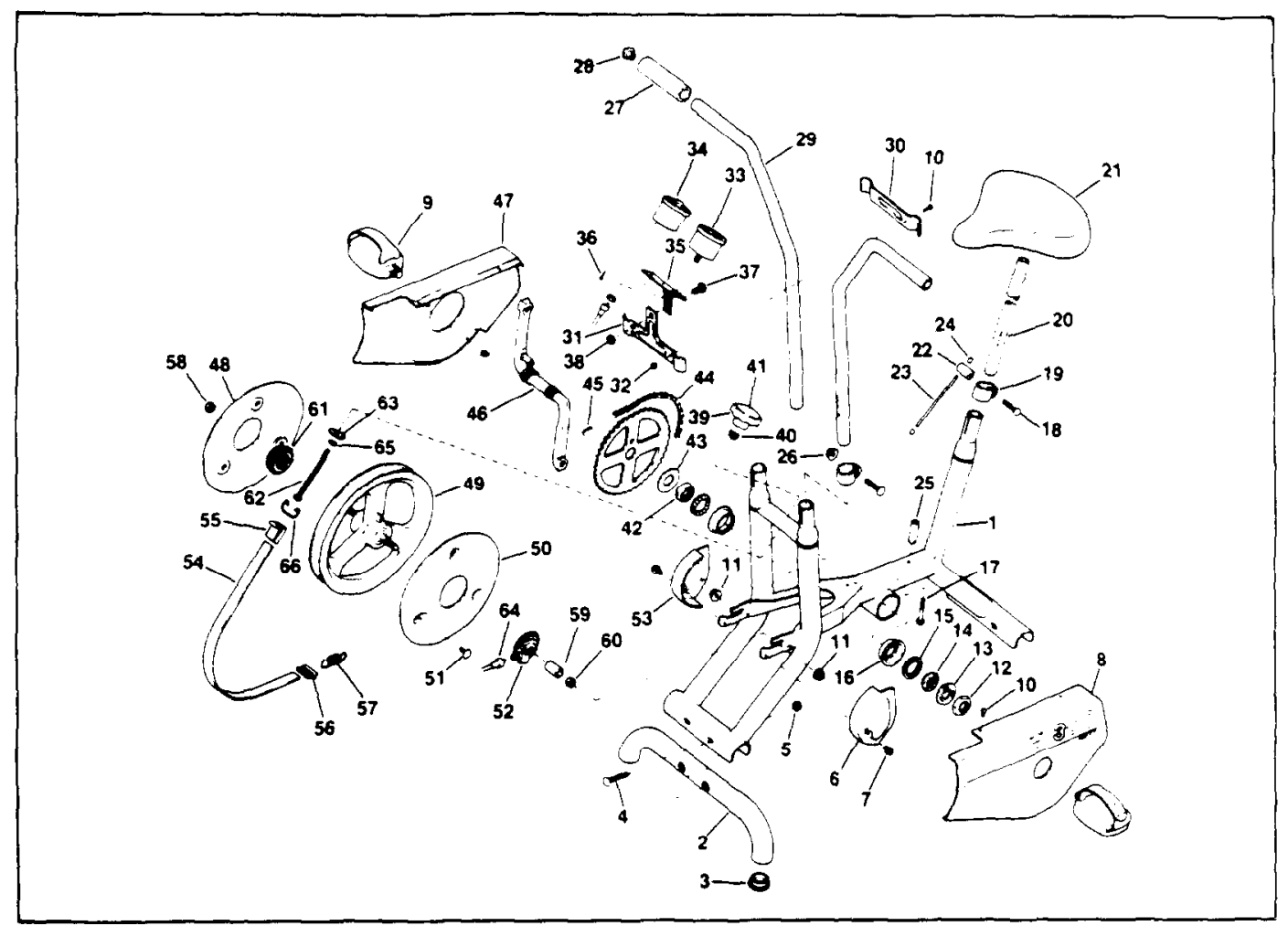

Fig. 2. Exploded assembly drawing of the fitness cycle (reprinted from the owner's manual of the Huffy 300 ).

responsible for assembling part of the fitness cycle as shown below:

$w=1$ Flywheel Subassembly,

$w=2 \quad$ Pedal and Crank Subassembly,

$w=3$ Chain and Chain Guards,

$w=4$ Tension Belt Subassembly,

$w=5$ Speedometer, Timer, and Cable,

$w=6$ Seat, Handle Bar, and Stabilizer Subassembly.

Each component shown in Table 1 is treated either as a primary component or a secondary component. All primary components are delivered to the shop floor either in component containers (with line stocking) or in kit containers. Only one component container (or stationary kit container, if any) of each type is stored at each workstation. All components such as fasteners, washers, spacers, plugs, etc., are considered secondary components and they are assumed to be bulk delivered to the shop floor in component containers. Consequently, we will not include secondary components in our analysis.

The workstation and other data are shown in Table 2 where the following information is displayed for each workstation type: (1) the assembly area required per workstation $\left(a_{w}\right)$, (2) the number of parallel workstations $\left(\pi_{w}\right),(3)$ the set of (primary) components used $\left(\Phi_{w^{\prime}}\right),(4)$ the number of pieces per component container for each primary component $\left(p_{s}\right)$, and (5) the set of secondary components used.

We assume that 320 fitness cycles must be assembled in an $8 \mathrm{~h}$ day; that is $D=320$ units/day or $1.5 \mathrm{~min} / \mathrm{unit}$. The number of parallel workstations shown in Table 2 are based on the assumption that the cycle time for workstations 1 and 2 is equal to 7.5 $\mathrm{min} / \mathrm{unit}$ while the cycle time for the remaining stations is equal to $4.5 \mathrm{~min} / \mathrm{unit}$. Remaining 
Table 1

Parts list for the fitness cycle (reprinted from the owner's manual of the Huffy 300 )

\begin{tabular}{|c|c|c|c|c|c|c|c|}
\hline $\begin{array}{l}\text { Key } \\
\text { No. }\end{array}$ & $\begin{array}{l}\text { Part } \\
\text { No. }\end{array}$ & Description & Qty. & $\begin{array}{l}\text { Key } \\
\text { No. }\end{array}$ & $\begin{array}{l}\text { Part } \\
\text { No. }\end{array}$ & Description & Qty. \\
\hline 1 & 900600 & Frame & 1 & 35 & 800895 & Bracket & 1 \\
\hline 2 & 800926 & Stabilizer & 2 & 36 & 203144 & Screw & 2 \\
\hline 3 & 207224 & Plug & 4 & 37 & 203168 & Bolt & 1 \\
\hline 4 & 203040 & Carriage Bolt & 4 & 38 & 203017 & Nut & 1 \\
\hline 5 & 203039 & Flange Nut & 4 & 39 & 207225 & Knob. Brake & 1 \\
\hline 6 & 207235 & Guard, Front LH & 1 & 40 & 203166 & Flange Nut, LH & 1 \\
\hline 7 & 203086 & Screw, Self Tap & 6 & 41 & 205965 & Label & 1 \\
\hline 8 & 900601 & Chain Guard, LH & 1 & 42 & 205072 & Cone & 1 \\
\hline 9 & 205032 & Pedals (Pair) & 1 & 43 & 800832 & Washer & 1 \\
\hline 10 & 203033 & Screw & 4 & 44 & 800923 & Chain & 1 \\
\hline 11 & 203032 & Flange Nut & 2 & 45 & 203513 & Connector Link & 1 \\
\hline 12 & 205071 & Hex Nut & 1 & 46 & 205053 & Crank & 1 \\
\hline 13 & 205075 & Washer & 1 & 47 & 900602 & Chain Guard, RH & 1 \\
\hline 14 & 205074 & Cone & 1 & 48 & 205126 & Freewheel & 1 \\
\hline 15 & 205069 & Bearing & 2 & 49 & 900604 & Wheel Assembly & 1 \\
\hline 16 & 205070 & Bearing Cup & 2 & 50 & 205441 & Shroud & 2 \\
\hline 17 & 203067 & Bolt, Hex Head & 1 & 51 & 205438 & Shroud Fastener & 6 \\
\hline 18 & 203132 & Carriage Bolt & 3 & 52 & 205426 & Speedometer Sending Unit & 1 \\
\hline 19 & 205063 & Post Clamp & 3 & 53 & 207234 & Guard, Front RH & 1 \\
\hline 20 & 800924 & Seat Post & 1 & 54 & 901268 & Strap Brake Kit & 1 \\
\hline 21 & 205010 & Seat & 1 & 55 & 207237 & Buckle & 1 \\
\hline 22 & 808806 & Nut. Quick Adjust & 1 & 56 & 207238 & Buckle & 1 \\
\hline 23 & 800854 & Pivot Shaft & 1 & 57 & 203726 & Spring & 1 \\
\hline 24 & 207223 & End Cap & 2 & 58 & 203015 & Nut. Thick & 1 \\
\hline 25 & 203162 & Spacer & 1 & 59 & 203165 & Spacer & 1 \\
\hline 26 & 203016 & Flange Nut & 2 & 60 & 203051 & Nut, Thin & 1 \\
\hline 27 & 206986 & Grip & 2 & 61 & 205126 & Sprocket Nut & 1 \\
\hline 28 & 206987 & Plug & 2 & 62 & 203169 & Bolt & 1 \\
\hline 29 & 800929 & Handlebar & 2 & 63 & 203170 & Washer & 1 \\
\hline 30 & 800930 & Bracket & 1 & 64 & 205427 & Cable & 1 \\
\hline 31 & 800932 & Clamp & 1 & 65 & 203222 & Washer, Nylon & 1 \\
\hline 32 & 203034 & Nut & 2 & 66 & 203703 & O-Ring & 1 \\
\hline 33 & 205436 & Speedometer & 1 & 67 & 205938 & Owner's Manual & 1 \\
\hline 34 & 205437 & Timer & 1 & & & & \\
\hline
\end{tabular}

assumptions for the numerical example are as follows:

(1) Both the component container and the kit container are assumed to require an area of $5^{\prime}$ by $5^{\prime}$ on the shop floor; that is, $F=f=25$ square feet.

(2) The average number of partially assembled fitness cycles in the system, $\bar{W}$, is equal to 42 units (including those at the workstations).

(3) Only one component or kit container of each required type is staged at a workstation; that is, $\alpha_{s w}=\beta_{k w}^{\prime}=1$.
Before we discuss a kitting scheme and present additional data, we will present the results obtained for line stocking. First, using Eq. (1) we determine the $c_{w}$ values as follows: $c_{1}=55, c_{2}=53, c_{3}=70, c_{4}=20$, $c_{5}=126$, and $c_{6}=34$ component containers per day (after rounding off to the nearest integer). Hence, from Eq. (2) $R=716$ operations/day. The number of component containers delivered to the shop floor is equal to 358 containers/day (from Eq. (4)), not including empty container return. 
Table 2

Workstation and other data for the fitness cycle

\begin{tabular}{|c|c|c|c|c|c|c|}
\hline & \multicolumn{6}{|c|}{ Workstation } \\
\hline & 1 & 2 & 3 & 4 & 5 & 6 \\
\hline $\begin{array}{l}\text { Assy. area } \\
\text { No. of WSs }\end{array}$ & $\begin{array}{r}30 \\
5\end{array}$ & $\begin{array}{r}25 \\
5\end{array}$ & $\begin{array}{r}25 \\
3\end{array}$ & $\begin{array}{r}25 \\
3\end{array}$ & $\begin{array}{r}35 \\
3\end{array}$ & $\begin{array}{r}25 \\
3\end{array}$ \\
\hline $\begin{array}{l}\text { Primary components } \\
\text { (SKU \#) }\end{array}$ & $\begin{array}{ll}48 & (30) \\
49 & (30) \\
50 & (30) \\
52 & (50) \\
61 & (50)\end{array}$ & $\begin{array}{l}14(40) \\
15(50) \\
16(50) \\
42(40) \\
46(30)\end{array}$ & $\begin{array}{rr}6 & (40) \\
8 & (20) \\
9 & (50) \\
44 & (35) \\
47 & (20) \\
53 & (40)\end{array}$ & $\begin{array}{ll}39 & (60) \\
54 & (60) \\
55 & (100) \\
56 & (100) \\
66 & (100)\end{array}$ & $\begin{aligned} 2 & (20) \\
19 & (50) \\
20 & (30) \\
21 & (20) \\
23 & (100) \\
27 & (50) \\
29 & (20)\end{aligned}$ & $\begin{array}{ll}30 & (100) \\
31 & (100) \\
33 & (30) \\
34 & (30) \\
35 & (100) \\
64 & (120)\end{array}$ \\
\hline $\begin{array}{l}\text { Secondary components } \\
\text { (SKU \#) }\end{array}$ & $\begin{array}{l}11 \\
51 \\
58 \\
59 \\
60\end{array}$ & $\begin{array}{l}12 \\
13 \\
43\end{array}$ & $\begin{array}{r}7 \\
17 \\
25 \\
45\end{array}$ & $\begin{array}{l}40 \\
41 \\
57 \\
62 \\
63 \\
65\end{array}$ & $\begin{array}{r}3 \\
4 \\
5 \\
18 \\
22 \\
24 \\
26 \\
28\end{array}$ & $\begin{array}{l}10 \\
32 \\
36 \\
37 \\
38\end{array}$ \\
\hline
\end{tabular}

Notes:

1. Numbers in parenthesis indicate the number of pieces per component container for the corresponding SKU.

2. The frame (SKU \# 1) is moved through each workstation type; therefore, it is not included in any component or kit container.

3. All secondary components are "bulk delivered" to the shop floor; they are not included in the analysis.

The space requirements on the shop floor are computed from Eqs. (6) and (7) as $A_{1}=755, \quad A_{2}=750, \quad A_{3}=525, \quad A_{4}=450$, $A_{5}=630, A_{6}=525$, and $A=3,655$ square feet. If we deduct the $a_{w}$ values for all the workstations, that is, if we deduct $\sum_{w} a_{w} \pi_{w}=605$ sq. ft. from the above total, the remaining space requirement $(3,050 \mathrm{sq}$. $\mathrm{ft}$.) reflects the space required to store $122 \mathrm{com}$ ponent containers (where one component container of each type is stored at each workstation). Lastly, the average WIP in number of pieces is computed from Eqs. (9) and (10) as $h_{1}=475, h_{2}=525, h_{3}=307.5$, $h_{4}=630, h_{5}=435, h_{6}=720$, and $H=3,092.5$ pieces.

For kitting we will consider two alternative plans, namely, plan A and plan B. Under plan $A$, all the (primary) components used at a workstation are delivered as a kit-no traveling kits are used. Under plan B, all the (primary) components are placed in a traveling kit which follows the frame of the fitness cycle - no stationary kits are staged at any workstation.

Further details on plan A are presented as follows. Recall that only one kit type (which contains all the primary components used at a workstation) is staged at each workstation. That is, $\Omega_{1}^{\prime}=\{1\}, \Omega_{2}^{\prime}=\{2\}, \ldots, \Omega_{6}^{\prime}=\{6\}$. Also, from Table 2 we have $S_{1}=\{48,49$, $50,52,61\}, S_{2}=\{14,15,16,42,46\}, \ldots$, $S_{6}=\{30,31,33,34,35,64\}$. The number of pieces of SKU $s$ used in kit type $k$, that is, $p_{s k}$, is equal to the number of pieces of SKU $s$ used in one fitness cycle, that is, $m_{s}$, for all $s$. This implies that $\dot{d}_{k}=1$ for all $k$.

We assume that $n_{1}=n_{2}=n_{3}=n_{5}=8$, $n_{4}=10$, and $n_{6}=16$, where $n_{k}$ is the number of kits of type $k$ placed in a kit container. We 
also assume that the number of kit containers per batch, $q_{k}$, is equal to one for all $k$. (This implies that $b_{k}=n_{k}$ for all $k$.) Lastly, recall that only one kit container is staged at each workstation (i.e., $\beta_{k w}^{\prime}=1$ ) and $f=25$ square feet. No traveling kits are used.

For kitting plan A we obtain the following results. Consider the first kit type which contains five SKUs as shown in Table 2. With $b_{1}=n_{1}=8$, we need to retrieve each component container $320 / 8=40$ times per day. Since we have five component types in this kit, we would retrieve (then store) 200 component containers per day to assemble all the kits of the first type. Repeating the same calculation for all the other kit types, from Eq. (3) we obtain $R=2400+358=2,758$ operations/day. From Eq. (5), the total number of (stationary) kit containers delivered to all the workstations is equal to $40+40+\cdots+20=212$ containers per day (not including empty container return).

The space requirements on the shop floor are computed from Eqs. (8) and (7) as $A_{1}=275, \quad A_{2}=250, \quad A_{3}=150, A_{4}=150$, $A_{5}=180, A_{6}=150$, and $A=1,155$ square feet. As before, if we deduct 605 sq. ft. to account for the space required by the workstations, the remaining space (550 sq. ft.) reflects the space required to store 22 kit containers, which is equal to the total number of workstations as one would expect under plan A. The average WIP for plan $A$ in number of pieces is computed from Eqs. (11) and (12) as $h_{1}=120, \quad h_{2}=140, \quad h_{3}=84, \quad h_{4}=75$, $h_{5}=144, h_{6}=144$, and $H=707$ pieces. Note that the cycle time at each workstation is at least 4.5 minutes per fitness cycle and each kit container holds at least 8 kits. With $d_{k}=1$, this implies that each kit container supports at least 8 fitness cycles, i.e., at least $8(4.5)=36$ minutes of production.

Consider next kitting plan B. Recall that under this plan no stationary kits are staged at the workstations. Instead, all the primary components required to produce one fitness cycle are placed in one traveling kit. Hence, $d_{k}=1$, $n_{k}=1$, and we have only one traveling kit type, i.e., $\Omega^{\prime \prime}=\{1\}, \beta_{1}^{\prime \prime}=1$, and $\left|S_{1}\right|=34$. One may argue that, since the traveling kit container used in plan B would have to be larger than the other component or stationary kit containers used in the previous calculations, the space requirements at each workstation, i.e., the $a_{w}$ values, would have to be increased. Although this is probably true, we will use the original $a_{w}$ values for comparison purposes.

For plan B we will assume that the traveling kits are assembled in batches of four, i.e., $b_{k}=4$. (Since $n_{k}=1$, we obtain $q_{k}=4$, i.e., each batch contains four traveling kit containers.) Given the above information, from Eq. (3) we obtain $R=[2(320)(34) / 4]+358$ $=5,798$ operations/day. Under plan $B$, no stationary kit containers are dispatched to the workstations, and the traveling kit travels along with the product. Hence, no container flow - in the manner in which we define it here-occurs under plan $B$.

Consider next the space requirements and average WIP under plan B. Since no stationary kits are staged at the workstations, we have $A_{w}-\pi_{w} a_{w}$ or $A_{1}=150, A_{2}=125, \ldots$, $\mathrm{A}_{6}=75$ sq. ft. Hence, the total space requirement is equal to 605 sq. ft. as computed earlier. We have not developed an expression for the average WIP at each workstation type. However, we may compute the average WIP for the system from Eq. (13) where $\bar{W}=42$ and $\beta_{1}^{\prime \prime} n_{1} \sum_{s \in S_{1}} p_{s 1}=43$. We obtain $H=903$ pieces for plan B. (Recall that this figure does not include the WIP generated by partially assembled fitness cycles; it reflects only the average WIP generated by the components in the traveling kit.)

The above results are summarized in Table 3 where certain tradc-offs can be clearly observed. As shown in Table 3 and Fig. 3a, there is a significant increase in the demand placed on the end-of-aisle storage/retrieval system when kitting is used instead of line stocking. Also, note that plan B generates more container storage and retrievals than plan A. This is primarily due to the fact that in plan B the kit batch size is relatively small and there are many components in one kit; i.e., the traveling kit. Increasing the kit batch size 
Table 3

Line stocking versus kitting: results obtained from the numerical example

\begin{tabular}{lccc}
\hline & $\begin{array}{l}\text { Line } \\
\text { stocking }\end{array}$ & $\begin{array}{l}\text { Kitting } \\
\text { plan A }\end{array}$ & $\begin{array}{l}\text { Kitting } \\
\text { plan B }\end{array}$ \\
\hline $\begin{array}{l}R, \text { container } S / R \\
\text { (operations/day) }\end{array}$ & 716 & 2,758 & 5,798 \\
$\begin{array}{l}\text { Container flow } \\
\text { (containers/day) }\end{array}$ & 358 & 212 & 0 \\
$\begin{array}{l}\text { Space requirement } \\
\text { square feet }\end{array}$ & 3,655 & 1,155 & 605 \\
$\quad$ No. of containers & 122 & 22 & 0 \\
$\begin{array}{l}\text { Average WIP } \\
\text { (no. of pieces) }\end{array}$ & 3,093 & 707 & 903 \\
\hline
\end{tabular}

Notes:

1. In computing $R$, each storage or retrieval is considered an "operation."

2. For line stocking, container flow represents the component containers delivered from the storage area to the shop floor. For kitting plan $A$, it represents the stationary kit containers delivered to the shop floor from the storage/kit assembly area. With kitting plan B, no stationary kits are delivered to the shop floor, and the traveling kit is handled along with the product, by definition.

3. The space requirement reflects the space required by the workstations themselves and the space required to stage component containers or stationary kit containers.

would reduce the workload imposed on the storage/retrieval system. However, this may require the user to store some of the kits before they can be assigned to a frame which is ready to be delivered to the shop floor.

It is instructive to note that, if the overall storage space requirement for the component containers and the time required to pull the necessary parts from a component container (once it is brought to the end of the aisle) are given, one can use the algorithm developed by Bozer and White [5] to estimate the number of workers (i.e., "kit assemblers") and storage/ retrieval devices required in the kit assembly area. (With end-of-aisle kit assembly, the worker stands at the end of the aisle assembling the kits. The component containers are brought to the end of the aisle by a dedicated "device" which retrieves and subsequently stores each component container.) Given the number of workers and storage/retrieval devices required for each alternative, one can express the results shown in Fig. $3 a$ in terms of cost in order to perform an economic analysis.

The container flow is shown in Table 3 and Fig. 3b. With line stocking, the material handling effort required to supply the workstations with the necessary components is significantly more than that required with kitting plan A. (Of course, this is true if there is no significant cost difference between moving a component container and a kit container.) Note that no material handling effort is imposed with kitting plan $B$ since under this plan there are no containers delivered to the workstations and the only traveling kit is handled along with the product. Comparing Figs. $3 a$ and $3 b$ it becomes quite clear that, with kitting, the material handling effort "shifts" from the shop floor to the storage/retrieval system in our example. Using our model and appropriate parameter values, one can determine the extent of this "shift" in other problems and perform an economic analysis if the appropriate cost data are made available.

In addition to reducing the handling effort on the shop floor, as seen from Table 3 as well as Figs. $3 \mathrm{c}$ and $3 \mathrm{~d}$, kitting results in floor space savings. The exact floor space requirements will vary according to the stacking patterns used for the component containers and the kit containers. (In our model we simply multiply the floor space requirement of each container with the number of containers.) Regardless of the exact floor space requirements, however, in our numerical example kitting clearly reduces the number of containers staged on the shop floor (see Fig. 3d).

The above reduction in containers can also be observed in Fig. 3e where a significant reduction in average WIP is obtained with kitting. Although the difference is not a significant one, note that kitting plan $B$ results in more WIP than kitting plan A. The exact difference in average WIP between the two plans is closely related to the average number of partially assembled products in the system. As 


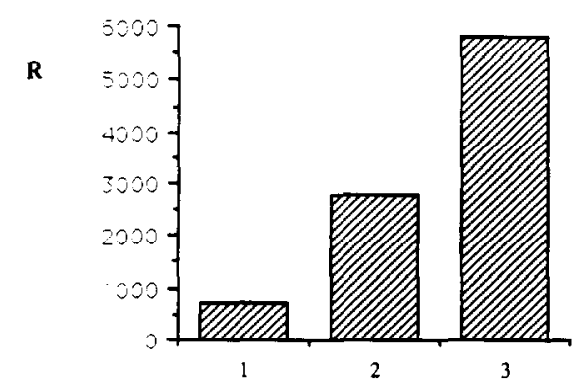

a) Container Storage and Retrieval

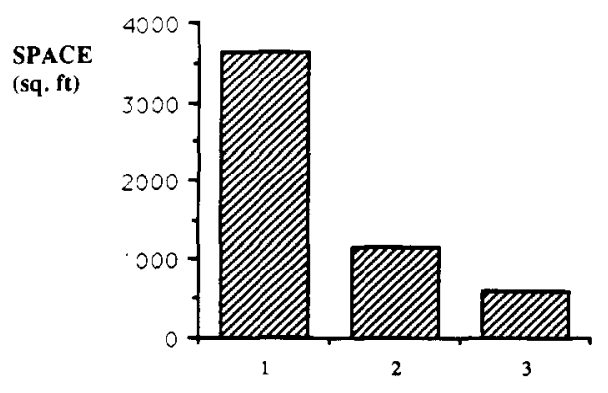

c) Floor Space Requirement (sq. ft)

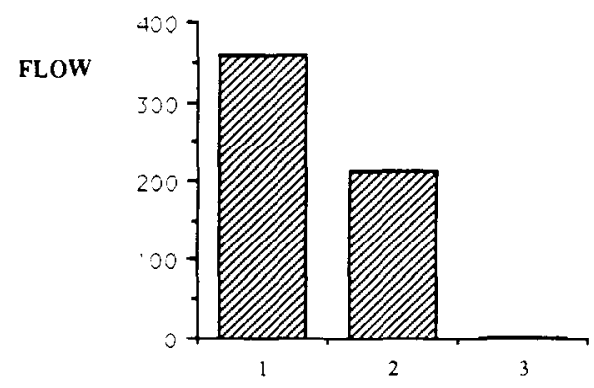

b) Container Flow

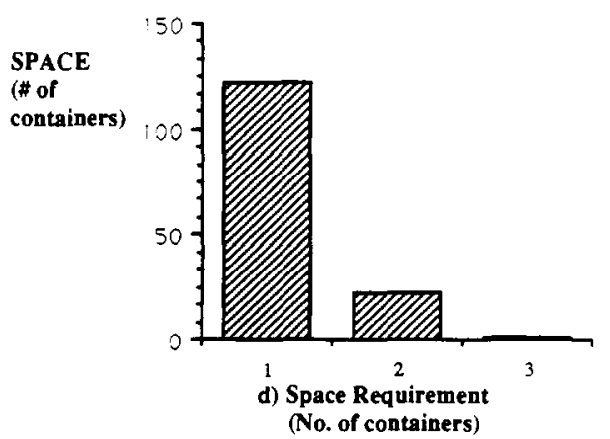

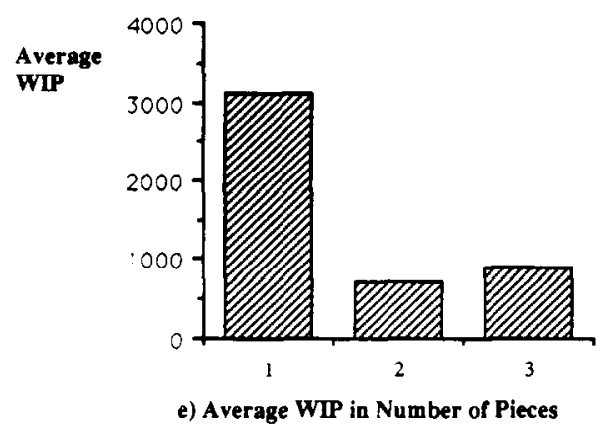

Fig. 3. Line stocking versus kitting: rcsults obtained from the numerical example: (1) Line stocking, (2) kitting plan $\Lambda$, (3) kitting plan B.

this number increases, the average WIP associated with kitting plan B will increase while it will remain the same with kitting plan A.

In summary, the results obtained from the example show certain numerical differences between kitting and line stocking. Although the results we obtained are somewhat predictable (for example, the survey results reported in Ref. [4] indicate that reducing in-process inventory is a top-ranked factor which motivated the use of kitting), we believe the model we developed as part of this study facilitates a quantitative comparison between line stocking and alternative kitting plans. Coupled with appropriate cost data and an economic model, the results obtained from our model (such as those shown in Table 3 and Fig. 3) can be used to select the most preferred system at least from the standpoint of material handling. (Reducing material handling was among the top five factors that motivated the use of kitting according to the survey results presented in Ref. [4].) We must also caution the reader that the numerical results we present here are 
based on specific parameter values and a realistic but hypothetical problem. Applying our model (or its possible extensions) to real-world problems will no doubt further improve our knowledge and insight on line stocking versus kitting.

\section{Conclusions and new research directions}

With this paper we hope to provide a common basis for conducting research on line stocking versus kitting and their impact on manufacturing. Based on the site visits we performed for this purpose, we developed formal definitions and presented a qualitative evaluation of kitting, including a list of advantages and limitations. Subsequently, we developed a descriptive model which should facilitate a quantitative comparison between various kitting plans and line stocking. Although the model is based on a number of assumptions (some of which are perhaps quite simplistic), we believe it can be quite useful in a preliminary decision stage. We also hope that it would serve as a "starting point" or "benchmark" for future models developed in this area.

The numerical example indicates that kitting tends to shift the material handling workload from the shop floor to the storage/ retrieval system. We also observed that shop floor space requirements and the average work-in-process (WIP) decreases with kitting. However, the direction as well as the magnitude of the above changes is closely related to the parameter values and the problem setting (i.e., type of product assembled, method used for assembling, storing, and handling the kits, and so on). Different results may be obtained with different parameter values for the same problem setting, or same parameter values for different problem settings. As long as the problem setting supports it, the model we propose here can be used with the appropriate parameter values to perform quantitative comparisons. Although we have not performed it, parametric analysis also seems possible with our model.
We must also stress that the model and the numerical example are based on just-in-time (JIT) kitting. That is, kits are assembled and delivered on an "as needed" basis. In contrast, if kits are assembled ahead of time and stored until they are ready to be used, the demand placed on the storage/retrieval system will further increase. As we mentioned earlier in the paper, although with a variable kit mix it may be difficult to implement JIT kitting, some other limitations of kitting becomes more evident if kits are not assembled on a JIT basis.

Obviously, our model can be enhanced in several ways. We hope these enhancements will provide opportunities for more research on kitting. Aside from the model, however, we believe there are some fundamental and new research directions which can be pursued in this area. The following are those which seem promising:

(1) Although in our model we assumed that the workstation cycle times do not change between line stocking and kitting, it is fairly straightforward to extend our model to capture a possible change in cycle times as long as the resulting shop configuration is given for both approaches. Since the assembly time required for each "elementary task" is likely to decrease with kitting, a more comprehensive comparison of the two concepts can be performed if the shop is configured (i.e., the "assembly line" or the "flow shop" is balanced) with and without kitting. Using the most appropriate shop configuration for each concept would amplify the differences between the two.

(2) The kit structure is mostly determined by the shop configuration. That is, the components assigned to stationary and traveling kits depend on which components are used at each workstation. However, the latter depends on the shop configuration since the tasks assigned to a particular workstation define the type and number of components used at that workstation. Given this interrelation between the shop configuration and the kit structure, we need to develop "line or shop balancing" 
algorithms with the resulting kit structure in mind.

(3) Based on the preliminary results presented here, there seems to be certain trade-ofls even within alternative kitting plans. Hence, before kitting is compared with line stocking, one must first determine the most desirable kitting plan. It appears that developing the kit structure is one of the critical steps. Part of this task involves the designation of each kit type as stationary or traveling, and the assignment of components to each kit type. Developing formal models to determine the "most efficient" kit structure for a given shop configuration seems essential at this point in time. Such models will also make it possible to incorporate the kit structure with "line or shop" balancing algorithms as discussed in the previous item.

(4) Aside from having ideal shop configurations which are likely to be different, another major difference between kitting and line stocking - which to our knowledge has not been explored in the research literature - is the impact the two concepts would have on the assembly (i.e., the shop) schedule. Since kitting is used quite often with small lot, multi-product assembly operations, the shop schedule plays a significant role in overall productivity. Recall that, with kitting, product changeovers are accomplished relatively easily. This is likely to have a significant impact on the "ideal" schedule. Hence, one could develop and evaluate "ideal" schedules with and without kitting for comparison purposes. In the process, one may also develop scheduling algorithms which explicitly take advantage of the flexibility offered by kitting. In doing so, one must not overlook the relationship between the kit batch size and the batch sizes used in the shop schedule.

(5) Container design seems to have a significant impact on both line stocking and kitting since it defines certain important parameters such as number of component pieces or number of kits per container. It also defines the floor space requirements on the shop floor and the storage space requirements in the storage/retrieval system. Container design is also closely related to the kit structure. If the component containers are designed such that multiple SKUs can be stored in each container, then one may minimize the necessary container retrievals to assemble a given kit. To our knowledge, container design and configuration from the above standpoint remains one of the ledst explored issues in the research literature.

(6) Kitting seems to offer certain advantages which may facilitate balancing "mixed model" assembly lines. To our knowledge, the impact of kitting on mixed model assembly lines has not been investigated in the research literature.

We believe the above issues related to kitting are challenging and relevant research topics which have immediate or potential applications in industry.

\section{Acknowledgements}

This study was partially supported by the Material Handling Research Center at Georgia Institute of Technology, the National Science Foundation under Grant ISI 8300 965, and Dr. Bozer's Presidential Young Investigator Award under NSF Grant DMC 8858562.

\section{References}

[1] Gupta, N.K., 1982. Kitting matrix adds accuracy to small part picking system. Ind. Eng., 14: 35-38.

[2] Anonymous, 1985. Mobile parts storage brings big benefits to kitting. Mater. Handling Eng., 40: 80-90.

[3] Wilhelm, W.E. and Wang, L., 1986. Management of component accumulation in small-lot assembly systems. J. Manufac. Sys., 5: 27-39.

[4] Sellers, C.J. and Nof, S.Y., 1986. Part kitting in robotic facilities. Mater. Flow, 3: 163-174.

[5] Bozer, Y.A. and White, J.A. Design and performance models for end-of-aisle order picking systems. Manage. Sci., 36: 852-866. 